\title{
Adaptive Window-Tuning Algorithm for Efficient Bandwidth Allocation on EPON
}

\author{
Sangho Lee, Tae-Jin Lee, Min Young Chung, and Hyunseung Choo* \\ School of Information and Communication Engineering, \\ Sungkyunkwan University, Korea \\ $\{$ ianlee,tjlee, mychung, choo\}@ece.skku.ac.kr
}

\begin{abstract}
Ethernet passive optical network (EPON) has been considered to solve the last mile bottleneck problem. In an effort to accommodate the explosive bandwidth demands from subscribers, the optical line terminal (OLT) efficiently divides and allocates time slots for data upstream to all optical network units (ONUs) in EPON. This technology is expected to be a core in the future fiber-to-the-home/-office/-curb (FTTH/O/C). We study previous algorithms for dynamic bandwidth allocation (DBA) in interleaved polling with adaptive cycle time (IPACT). For effective bandwidth allocation of the uplink channel, we propose an adaptive window-tuning algorithm (AdWin) based on the excessive bandwidth. This algorithm not only satisfies bandwidth demands of ONUs within the possible scope, but also seeks fairness among ONUs. The comprehensive computer simulation results indicate that the proposed scheme is up to $94 \%$ and $94 \%$ lower than previous schemes in terms of average packet delay and average queue size, respectively. It also demonstrates up to $86 \%$ improved performance in regards to packet loss ratio.
\end{abstract}

\section{Introduction}

Ethernet passive optical network (EPON) is an emerging solution to mitigate the last mile [1 bottleneck problem between backbone and access networks connected with business and residential subscribers [2]. In order to accommodate the huge demands of subscribers resulting from the explosive growth of the Internet and numerous high-broadband applications, many studies 124] on EPON have been discussed. In general, an EPON architecture based on tree topology is a point-to-multipoint fiber optical network, which consists of an optical line terminal (OLT), a 1:N passive star coupler (or splitter/combiner) 2, and multiple optical network units (ONUs) which share an optical fiber between the passive star coupler and the OLT for data upstreaming [2].

Previously, in order to efficiently manage data upstreaming, various DBA schemes have been studied. Interleaved polling with adaptive cycle time (IPACT) [3] is a typical scheme, where several disciplines such as limited, constant credit,

\footnotetext{
^ Corresponding author.
} 
linear credit and elastic services are proposed to prevent monopolizing the entire bandwidth.

Limited service grants the requested bandwidth, but imposes the limit of the transmission window. Although it is the most conservative, it has the best performance of DBA disciplines in IPACT. Based on limited service, constant and linear credit services are proposed [3]. Basically, these services take into account newly arriving packets from subscribers during the interval between the current upstream end time and the next upstream start time of a particular ONU 34 . The constant credit service allocates the requested bandwidth and fixed credits as additional bandwidth. Linear credit service is equivalent to the constant credit service other than how to add credits. It decides proportionally additional bandwidth based on the requested bandwidth. However, both services waste bandwidth if not used. Meanwhile, elastic service allocates bandwidth to the current ONU based on the past $N$ grants where $N$ is the number of ONUs. It is an attempt to remove the transmission window limit. It is imposed only on the maximum poll cycle time 3 .

However, these services do not necessarily serve bandwidth to every ONU fairly and efficiently, because in case of limited service, OLT grants bandwidth to every $\mathrm{ONU}$ as much as the maximum window limit regardless of requested bandwidth under heavy traffic load. It can lead to relative unfairness among ONUs. In the meantime, elastic service can grant entire bandwidth within a cycle time to an ONU. Nevertheless, after granting considerable bandwidth, OLT cannot allocate enough bandwidth to the next ONUs on account of the maximum polling cycle time. It also brings about another fairness problem.

In this paper, an adaptive window-tuning algorithm (AdWin) is proposed to resolve the problems in IPACT disciplines, which suffer from unfairness and, in some cases, inefficiency. It allows OLT to efficiently allocate bandwidth to all ONUs, by changing the limits of the transmission window (time slot) [2]. The variable transmission window limit exerts to assign excessive bandwidth to the ONUs that have greater demands. This algorithm reduces not only average packet delay, but also average queue size and supports better services than previous schemes. In accordance with comprehensive computer simulation results, the proposed scheme has up to $94 \%$ and $94 \%$ lower average packet delay and average queue size, respectively. The scheme also results in up to $86 \%$ better performance in terms of packet drop ratio.

The remainder of the paper is organized as follows. In Section 2, the AdWin is proposed as a promising solution. Next in Section 3 we evaluate the performance of the proposed scheme, in terms of the average packet delay, average queue size, and packet loss ratio. Section 4 concludes this paper.

\section{An Adaptive Window-Tuning Algorithm}

The proposed algorithm, AdWin, is a generous bandwidth allocation scheme that utilizes the remaining bandwidth in the past. The remainder bandwidth is used to increase the transmission window limits for the subsequent $N$ ONUs. 
To calculate the remainder bandwidth, a limit threshold is needed first, this is obtained from the maximum window size in IPACT [23. For the difference between the limit threshold and the granted bandwidth for the ONU $i-1$, the remainder bandwidth is decided and equally divided by the number of ONUs. The divided remainder bandwidth is then added to the limit of transmission window for the ONU $i$. In doing so, the transmission window limit for the subsequent $N$ ONUs (ONU $i$ to $i-1$ ) is determined. However, the remainder bandwidth may be negative when the granted bandwidth for the ONU $i-1$ is greater than that of the limit threshold. In this case, the over-granted bandwidth is equally divided and added to the previously determined limit of the transmission window, as in the case of the remainder occurrence. Thus, the limit for the next ONUs changes every bandwidth granting.

Due to the increasing limit, ONUs have greater possibility of bandwidth grants than that with the limit threshold. Therefore, they can accommodate bandwidth demands accordingly. The proposed AdWin algorithm is based on the limited service in IPACT. When it comes to granting bandwidth, the OLT compares the bandwidth request to the decided limit of the transmission window. This is similar to the limited service, which is presented as follows: $G^{[i]}=\min \left(R^{[i]}, W_{M A X}^{[i]}\right)$, where $G^{[i]}$ is granted bandwidth, $R^{[i]}$ is requested bandwidth, and $W_{M A X}^{[i]}$ is the maximum window size for ONU $i$.

\section{Performance Evaluations}

To evaluate performance of the DBA schemes, we consider an EPON system consisting of an OLT and 16 ONUs with interleaved polling operation. The simulation parameters are determined as in 3. The traffic pattern on access networks is characterized in accordance with self-similarity and long-range dependence (LRD) 35].

Fig. 1 presents average packet delay, average queue size, and packet loss ratio of limited, elastic services, and AdWin algorithm, under the traffic load $\rho$, from 0.05 to 0.95 . In the region of $0.45 \leq \rho \leq 0.60$, the packet delay drastically increases as $\rho$ increases, but its value among all the considered schemes is different, because the average granted bandwidth for ONUs is different. In addition, the average queue size shows a similar trend to the average packet delay. From the results, the proposed scheme outperforms the limited and elastic services for the traffic load $\rho$ in $[0.45,0.60]$. The AdWin has up to $94(94) \%$ and $91(90) \%$ lower average packet delay (average queue size) than that of limited and elastic services, respectively.

The packet loss ratio for the considered algorithms is shown in Fig. 1(c), Due to drastic increases in the average buffer size of ONUs, compared with limited and elastic services, the AdWin has a lower packet loss ratio for $0.55 \leq \rho<0.60$. Especially, for $\rho=0.55$, the AdWin has no packet loss and for $\rho=0.575$, it is $86 \%$ and $69 \%$ lower than that of limited and elastic services, respectively. 


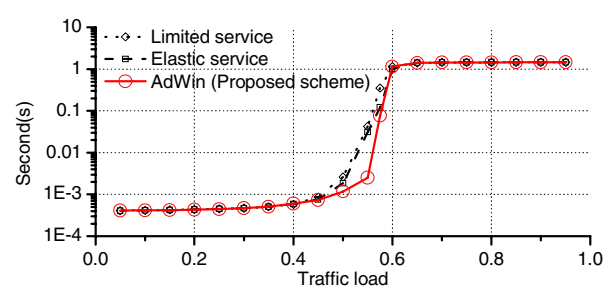

(a) Average packet delay

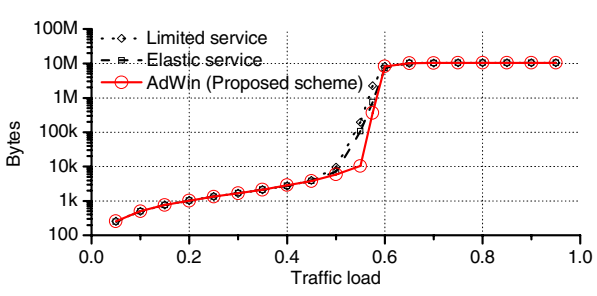

(b) Average queue size

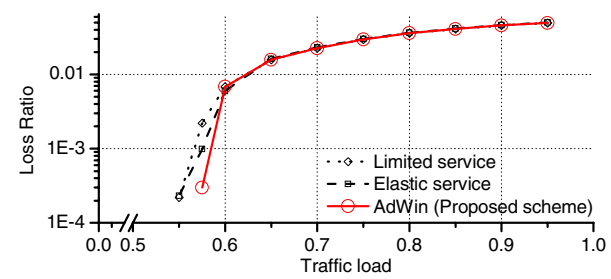

(c) Packet loss ratio

Fig. 1. Performance comparison among considered schemes

\section{Conclusion}

In order to enhance the performance of EPON system, we propose AdWin which alters transmission window limits based on past information of bandwidth allocation. It allocates the bandwidth to all ONUs efficiently and fairly. Consequently, the EPON system with AdWin algorithm can provide high-quality services for end users on last mile.

Acknowledgments. This research was supported by MIC, Korea under ITRC IITA-2006-(C1090-0603-0046) and the Korea Research Foundation Grant funded by the Korean Government(MOEHRD) (KRF-2005-042-D00248).

\section{References}

1. Kramer, G. and Pesavento, G.: Ethernet Passive Optical Network (EPON): Building a Next-Generation Optical Access Network. IEEE Communications Magazine Vol. 40, (2002) 66-73

2. Zheng, J. and Mouftah, H.T.: Media Access Control for Ethernet Passive Optical Networks: An Overview, IEEE Communications Magazine Vol. 43, (2005) 145-150

3. Kramer, G., Mukherjee, B., and Pesavento, G.: Interleaved Polling with Adaptive Cycle Time (IPACT): A Dynamic Bandwidth Distribution Scheme in an Optical Access Network, Photonic Network Communications Magazine, Vol. 4, (2002) 89107

4. Byun, H., Nho, J., and Lim, J.: Dynamic Bandwidth Allocation Algorithm in Ethernet Passive Optical Networks, Electronics Letters, Vol. 39, (2003) 1001-1002

5. Leland, W.E., Taqqu, M.S., Willinger, W., and Wilson, D.V.: On the Self-Similar Nature of Ethernet Traffic (Extended Version), IEEE/ACM Transation on Networking, Vol. 2, (1994) 1-15 